\title{
Optomechanical microwave oscillator and frequency comb generation in a full phononic bandgap 1D optomechanical crystal cavity
}

\author{
Laura Mércade ${ }^{1, *}$, Leopoldo L. Martin², and Amadeu Griol ${ }^{3}$,Daniel Navarro-Urrios ${ }^{3}$,Alejandro Martínez ${ }^{3}$, \\ ${ }^{1}$ Nanophotonics Technology Center, Universitat Politècnica de València, Camino de Vera s/n, 46022 Valencia, Spain \\ ${ }^{2}$ Departamento de Física, Facultad de Ciencias, Universidad de la Laguna \\ ${ }^{3}$ MIND-IN2UB, Departament dEnginyeria Electrònica i Biomèdica, Facultat de Física, Universitat de Barcelona, Martí Franquès 1, \\ 08028 Barcelona, Spain
}

\begin{abstract}
In this work we show that a silicon optomechanical crystal cavity can be used as an optomechanical oscillator when driven to the phonon lasing condition with a blue-detuned laser. The optomechanical cavity is designed to have a breathing like mode vibrating at $\Omega_{m} / 2 \pi=3.897 \mathrm{GHz}$ in a full phononic bandgap. Our measurements show that the first harmonic displays a phase noise of $-100 \mathrm{dBc} / \mathrm{Hz}$ at $100 \mathrm{kHz}$. Stronger bluedetuned driving leads eventually to the formation of an optomechanical frequency comb, with lines spaced by the mechanical frequency. The measured phase noise grows up with the harmonic number, as in classical harmonic mixing. We present real-time measurements of the comb waveform and show that it can be adjusted to a theoretical model recently presented. Our results suggest that silicon optomechanical cavities can play a role in integrated microwave photonics.
\end{abstract}

\section{Introduction}

Cavity optomechanics studies the interaction between light and sound waves simultaneously confined in a cavity [1]. In an optomechanical cavity (OMC), confined mechanical waves can coherently modulate an optical signal at $\mathrm{MHz}$ and even $\mathrm{GHz}$ frequencies via optomechanical interaction, becoming relevant in microwave photonics. Furthermore, since OMCs are nonlinear elements, multiple harmonics of the fundamental mechanical vibrations can be overimposed on the optical signal [2], a phenomenon that has been interpreted theoretically as an optomechanical frequency comb (OFC) [3].

\section{Optomechanical frequency comb}

The OMC that we exploit here consist in a suspended silicon nanobeam with one-dimensional (1D) periodicity [4]. The key idea is to have OM mirrors on each nanobeam side whilst optical and mechanical resonant modes are confined in the central region, having a good overlap between them. Our structure results of an optical mode at $\lambda_{r}=(1522.3 \pm 0.3) \mathrm{nm}$ with an optical quality factor of $Q_{o}=5 \times 10^{3}$, presented in Fig. 1(a), and a mechanical mode at $\Omega_{m} / 2 \pi=3.897 \mathrm{GHz}$ with an optomechanical coupling rate of $g_{0} / 2 \pi=(660 \pm 70) \mathrm{kHz}$, depicted in Fig. 1(b). In addition, we have designed our structure in order to have a full phononic bandgap to reduce the phonon leakage. A simulation, in Fig. 1(c), of the phononic band diagram from the profile extracted from a Scanning Electron Microscopy (SEM) image, which is shown (see Fig.

*e-mail: laumermo@ntc.upv.es 1(d)), shows that the experimental mechanical modes lies into the total bandgap.

The mechanical mode can be driven to the phonon lasing regime under proper conditions. When driving the OMC with a blue-detuned laser with respect to the optical resonance at even higher power, higher-order harmonics can be observed in the detected signal. This closely resembles an optical frequency comb (OFC) of OM nature, recently analyzed theoretically in [3]. We measured up to the fifth harmonic, as can be seen in the optical spectrum of the generated OFC in Fig. 1(e). The theoretical dynamics generation[3] shows a good agreement with the acquired experimental optical traces in Fig. 1(f).

\section{Optomechanical oscillator}

High-quality microwave sources, which are required for a number of applications, are typically made by applying frequency multiplication to an electronic source. Amongst the different techniques to build an optoelectronic oscillator, we propose one obtained from an OM cavity when pumped with a blue-detuned laser source. In this case, since the involved mechanism is a self-sustained oscillation originated from OM interaction, from now on we will refer to it as an optomechanical oscillator (OMO). We made this experiment with the cavity proposed in the last section and we measured. For the first harmonic in Fig.2(a), the resulting noise figure evolution is presented in Fig. 2(c). For this harmonic the noise figure becomes as low as $(-100 \pm 1) \mathrm{dBc} / \mathrm{Hz}$ at $100 \mathrm{kHz}$, which is a remarkable good value for an $\mathrm{OEO}$ oscillating at $\mathrm{GHz}$ frequencies. We 

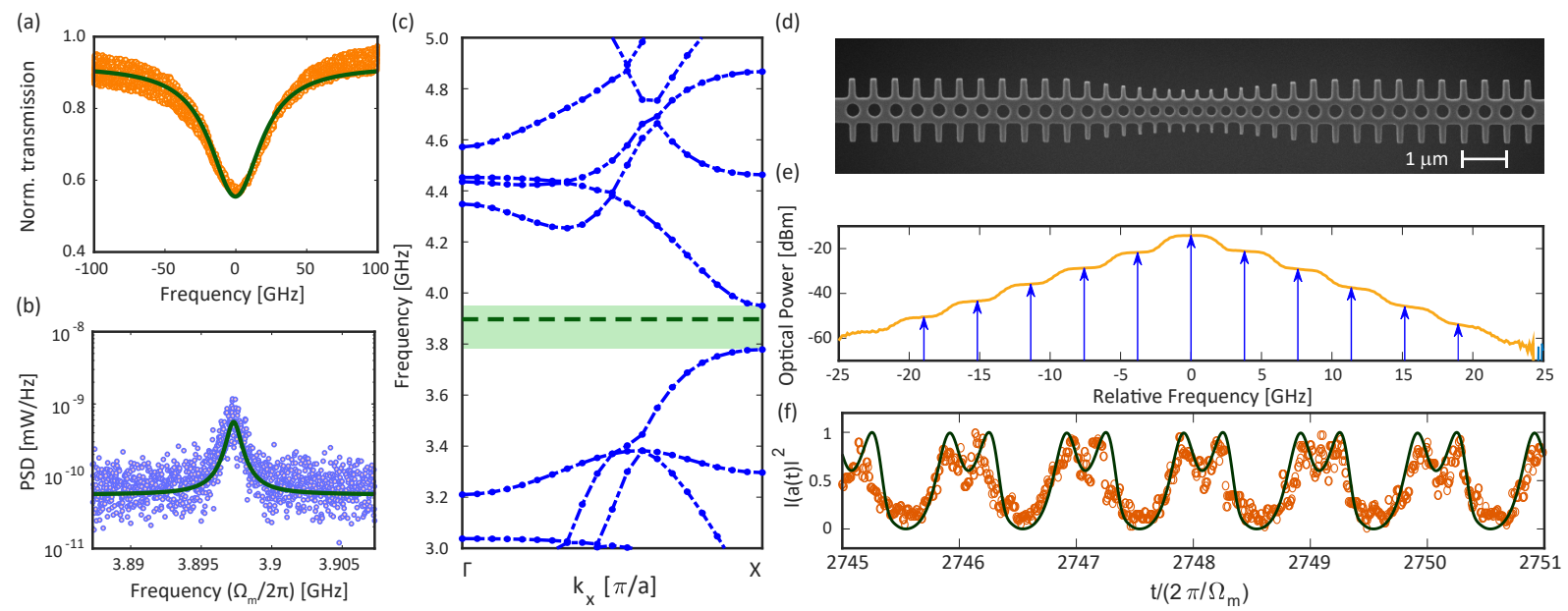

Figure 1. (a) Optical experimental resonance trace (purple) and Lorenztian fit (dark line). (b) Power spectral density of the mechanical resonance confined in the total bandgap. (c) Phononic band diagram for the real profile mirror unit cell extracted from the SEM image, including the experimental measured frequency of the mechanical mode (dashed line). (d) SEM image of the fabricated OM cavity. (e) Recorded optical spectrum in purple showing a set of peaks corresponding to different harmonics represented in blue at the expected position. (f) Comparison of the experimental (dots) and the theoretical (solid line) temporal OM-generated OFC traces.

have also measured the phase noise of the different harmonics, shown in 2(b), where the result is depicted in Fig. 2(d). In principle, the harmonic mixing process will result in an added phase noise of $20 \times \log (m)$ with respect to that of the first harmonic, which is well satisfied in our device.
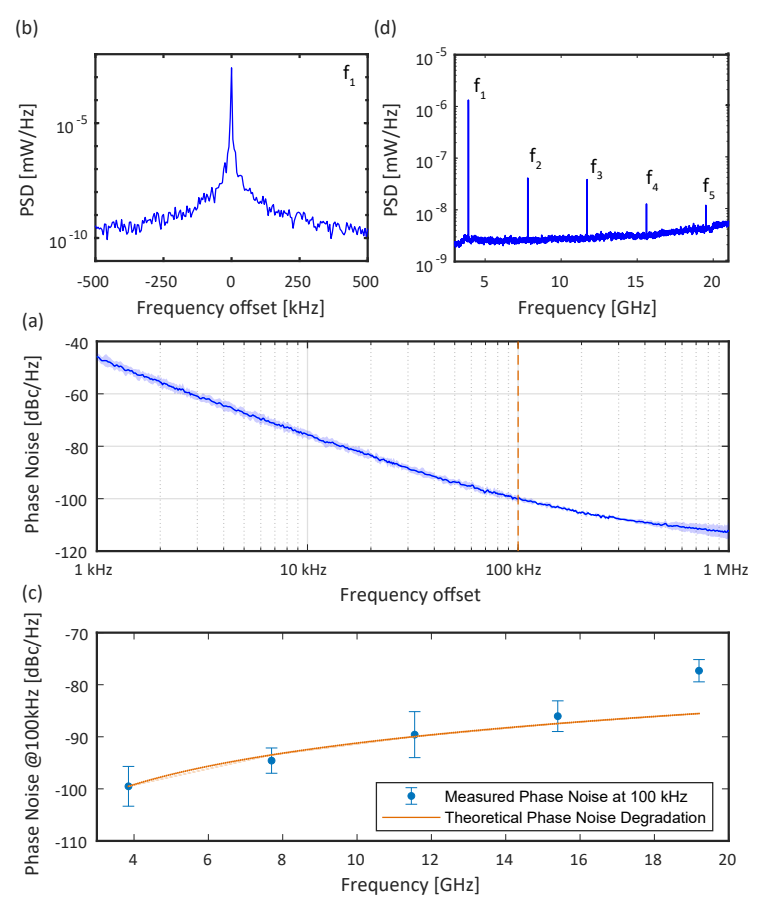

Figure 2. (a) Linear scale of the first harmonic centered at 3.87 GHz. (b) RF spectra of the generated OFC. (c) Mean phase noise of the generated microwave tone. (d) Phase noise of the different harmonics of the OFC with the theoretical phase noise degradation with respect to that of the 1 st harmonic.

\section{Conclusions}

In summary, we have demonstrated a new silicon-chip OMC with a large $\mathrm{OM}$ coupling rate for a $\mathrm{GHz}$ mode within a full phononic bandgap, which can perform as an ultracompact OMO. Operation at cryogenic temperatures would improve the phase noise [5] as a result of the enhancement of the mechanical $Q$ factor because of the full phononic bandgap. In addition, the preliminary demonstration of the OFC paves the way towards synthesis of microwave signals beyond the generation of pure $\mathrm{cw}$ tones. The main advantages of the OMC approach for microwave signal processing are its extreme compactness and low weight, highly desirable in space and satellite applications, and its compatibility with silicon electronics and photonics technology.

\section{References}

[1] M. Aspelmeyer et al., "Cavity optomechanics," Rev. Mod. Phys., vol. 86, pp. 1391-1452, 2014.

[2] M. Hossein-Zadeh and K. J. Vahala, "Photonic RF Down-Converter Based on Optomechanical Oscillation,” IEEE Photonics Tech. L., vol. 20, pp. 234-236, 2008.

[3] M. A. Miri et al., "Optomechanical frequency combs," New J. Phys., vol. 20, 043013, 2018.

[4] L. Mercadé et al., "Microwave generation and frequency comb in a silicon optomechanical cavity with a full phononic bandgap," arXiv:1912.06692 [physics.optics]

[5] M. Hossein-Zadeh and K. J. Vahala, "An Optomechanical Oscillator on a Silicon Chip," IEEE J. Sel. Top. Quant., vol. 16, pp. 276-287, 2010. 